\title{
Antropologia medica nella crisi ambientale. Determinanti biosociali, politica e campi di causazione
}

Medical anthropology in the environmental crisis. Biosocial determinants, politics and causation fields

\section{Elisa Pasquarelli e Andrea Filippo Ravenda}

\section{OpenEdition \\ Journals}

Edizione digitale

URL: http://journals.openedition.org/aam/2507

DOI: $10.4000 /$ aam.2507

ISSN: 2038-3215

\section{Editore}

Dipartimento Culture e Società - Università di Palermo

\section{Notizia bibliografica digitale}

Elisa Pasquarelli e Andrea Filippo Ravenda, «Antropologia medica nella crisi ambientale. Determinanti biosociali, politica e campi di causazione», Archivio antropologico mediterraneo [Online], Anno XXIII, n. 22 (1) | 2020, online dal 20 juin 2020, consultato il 27 janvier 2021. URL: http:// journals.openedition.org/aam/2507; DOI: https://doi.org/10.4000/aam.2507

Questo documento è stato generato automaticamente il 27 janvier 2021.

\section{c) (†)}

Archivio antropologico mediterraneo è distribuita con Licenza Creative Commons Attribuzione - Non commerciale - Non opere derivate 4.0 Internazionale. 


\title{
Antropologia medica nella crisi ambientale. Determinanti biosociali, politica e campi di causazione
}

\author{
Medical anthropology in the environmental crisis. Biosocial determinants, \\ politics and causation fields
}

Elisa Pasquarelli e Andrea Filippo Ravenda

\section{Introduzione ${ }^{1}$}

1 I danni ambientali provocati da attività industriali inquinanti e il loro impatto sulla salvaguardia e sull'integrità degli spazi fisici ed ecologici di vita e di lavoro sono stati fatti oggetto di interesse analitico da parte dell'antropologia medica italiana in tempi relativamente recenti. Il più ampio dibattito internazionale è stato avviato già negli anni Ottanta del secolo scorso dai lavori, fra altri, di Hans Baer, Merrill Singer, John Johnsen (1986), Ulrich Beck (1987), Martha Balshem (1993) e Adriana Petryna (1998) ${ }^{2}$. Tra la fine degli anni Settanta e gli anni Ottanta, infatti, il verificarsi di disastri che coinvolsero stabilimenti industriali e di produzione energetica - il disastro di Seveso (1976), l'esplosione dell'impianto P2T del petrolchimico di Brindisi (1977), il disastro di Bhopal (1984), quello di Chernobyl $(1986)^{3}$ - ha sicuramente stimolato una prima presa di coscienza da parte dell'opinione pubblica della pericolosità degli insediamenti industriali e dei rischi biologici connessi all'esposizione quotidiana ad agenti inquinanti. Negli Stati Uniti questo fenomeno è stato esaminato dal punto di vista antropologico quasi fin dal suo emergere all'attenzione generale, ispirando prospettive teorico-metodologiche innovative di comprensione e interpretazione critica delle connessioni tra le emissioni inquinanti, la salute e l'ambiente. Diversamente, in Italia, nonostante gli episodi di Seveso e di Brindisi abbiano stimolato dibattiti in ambito politico-sanitario, in quegli anni non sono state prodotte ricerche etnografiche su questo argomento. Soltanto nell'ultimo decennio sono state poi condotte valide etnografie - alcune delle quali ospitate in questa rivista - che inaugurano un 
promettente campo di riflessione critica sul rapporto tra inquinamento e rischio sanitario nel nostro Paese (Alliegro 2012; Ravenda 2014; Benadusi, Revet 2016), inserendosi nella cornice epistemologica del panorama internazionale. Potremmo dire che in Italia l'interesse etnografico per questo tema si manifesti proprio all'apice della crisi dei modelli di sviluppo industriale, con le relative drammatiche conseguenze occupazionali (perdita di posti di lavoro), sanitarie (aumento di patologie correlate plausibilmente all'inquinamento industriale) e ambientali (aree dismesse contaminate e abbandonate).

2 Il dibattito antropologico contemporaneo ha messo in evidenza i molteplici aspetti che, di volta in volta, associano o dissociano le emissioni inquinanti prodotte nelle aree industriali con la diffusione di patologie. Si tratta di affrontare questioni costantemente interrelate dentro campi di forza in cui le causalità sono il prodotto delle tensioni conflittuali tra attori sociali variamente posizionati e motivati da interessi spesso divergenti. Le questioni che sono in gioco riguardano contemporaneamente spazi scientifici, giuridici, politici, patrimoniali, sanitari all'interno dei quali prendono forma situate e mutevoli dinamiche di contrapposizione e negoziazione.

3 Il secondo convegno nazionale della Società Italiana di Antropologia Medica (SIAM), intitolato Un'antropologia per capire, per agire, per impegnarsi. La lezione di Tullio Seppilli, che si è tenuto a Perugia dal 14 al 16 giugno $2018^{4}$, ha offerto un'occasione di confronto sulla tematica dell'inquinamento con un focus principale sull'Italia e, in particolar modo, sui processi di industrializzazione che, tra la fine degli anni Cinquanta e l'inizio degli anni Sessanta, hanno coinvolto molte aree del Paese e del Mezzogiorno. Essi hanno determinato trasformazioni negli equilibri sociodemografici le quali sono ambiguamente ricollegabili, da un lato, a progettualità di sviluppo dei territori e, dall'altro, ad attività di sfruttamento intensivo di quegli stessi territori, che hanno inciso pesantemente sull'ambiente e sulla salute delle persone. I contributi della sessione Salute, ambiente, rischio hanno messo in luce che in simili contesti il diritto alla salute è stato sistematicamente eroso, costituendosi come spazio di una continua rivendicazione fisico-politica (Pizza, Ravenda 2012) in cui l'individuazione, la prova e la quantificazione del danno biologico subito dagli ambienti e dai corpi diventano fattori costitutivi, non di rado controversi e conflittuali, di nuove strategie per la salute e di nuove forme di cittadinanza e di partecipazione politica.

Comune cornice del Convegno è stato l'insegnamento di Tullio Seppilli che già negli anni Sessanta del secolo scorso, in continuità con la tradizione storicista gramsciana $\mathrm{e}$ demartiniana, sottolineava l'inscindibilità dei processi biologici e dei processi sociali nella condizione umana: «non soltanto una giustapposizione dei due tipi di leggi ma una intersezione» (Seppilli 1968: 242). Un concetto, questo, che Seppilli ha ribadito costantemente nella sua lunga vita professionale (Seppilli 1996, 2014; cfr. Seppilli 2008a, 2008b) e che ormai da tempo è stato recepito dall'antropologia medica contemporanea (Hahn, Inhorn 2009; Lock, Nguyen 2010; Biehl, Petryna 2013), nella cui circoscrizione epistemologica centrale è il riconoscimento del carattere storico-politico dei processi corporei e della capacità corporea di produzione e azione simbolica e storico-politica.

Come i contributi di questa sezione monografica di Archivio Antropologico Mediterraneo dimostrano, a fronte di ricerche etnografiche di lungo periodo, questioni cruciali investono i rapporti tra agenti patogeni, modelli di rischio e salute/malattia (Ravenda 2018); apparati normativi, pluralismo legale e giudizializzazione del diritto alla salute (judicialization of the right to health) (Biehl 2013); valutazione del rischio e del 
danno ambientale, autorità scientifica e movimenti per la giustizia ambientale (Waldman 2011); ricerca scientifica e azione politica (Boudia, Jas 2014). Fil rouge di tutte queste componenti co-agenti dei campi di forza coinvolti è l'intersezione tra determinanti biologiche e determinanti storiche, socioculturali e politiche dell'esperienza vissuta che «si integrano e si incorporano in forme anche molto complesse» (Seppilli 2014: 20).

\section{Antropologia medica e biomedicina}

6 Nella storia degli studi dell'antropologia medica (Ackerknecht 1971; Foster, Anderson 1978; Augé, Herzlich 1986; Atkinson 1987), la biomedicina è assurta a oggetto di osservazione e analisi dopo una prima fase dominata da un'attenzione precipua per le cosiddette "etnomedicine" e per le medicine tradizionali e popolari, nonché da un processo ermeneutico e analitico in cui la "medicina scientifica", di matrice occidentale, era utilizzata come una sorta di "lente di riferimento". Categorie quali corpo, corporeità, salute/malattia e i meccanismi terapeutici propri della biomedicina erano assunti dagli stessi antropologi come dati naturali e universali, e i presupposti oggettivati e oggettivanti biomedici come termini di paragone esplicativi nella lettura delle pratiche simboliche e materiali relative al dominio della salute e della cura in contesti etnologici.

7 Questo momento della tradizione disciplinare generale è stato definito da Tullio Seppilli (2014) «primo snodo» del processo trasformativo e di sviluppo epistemologico dell'antropologia medica italiana. Vi coesistevano due direttrici dominanti. L'una, avviatasi fra fine Ottocento e inizio Novecento in pieno clima positivista, poi culminata negli studi di tradizione teorico-metodologica gramsciana e nell'opera di Ernesto de Martino in Lucania (1958) e Salento (1961); l'altra, successiva agli anni Cinquanta, vocata all'inaugurazione di «un ambito di ricerche e progettualità operative concernenti le strategie e le pratiche delle istituzioni sanitarie ufficiali, i loro fondamenti teorico-pratici e i loro rapporti con le popolazioni» (Seppilli 2014: 19). È il «secondo snodo» che segna l'attrazione della biomedicina nella sfera delle riflessioni dell'antropologia medica, esponendola a un'interpretazione e critica storico-culturale che ne hanno poi svelato il carattere multiforme, cangiante, internamente diversificato, sottraendola all'identificazione con un sistema unitario, razionale, universale in quanto scientificamente fondato. Essa è invero prodotto dell'ambiente storico-culturale e politico nel quale è praticata, è influenzata da fattori socioeconomici, interessi accademici e finanziari, direttive politico-sanitarie, assunti ideologici e filosofici, aspetti che tende a espungere dalle retoriche della sua autorappresentazione (Pasquarelli 2008, 2018)5. Nel gergo dell'antropologia medica, biomedicina - e non "medicina" - proprio per evidenziarne la concentrazione preminente sugli aspetti biologici, cioè il riduzionismo biologico del suo approccio conoscitivo e operativo che limita i processi di salute e malattia «alla loro componente naturalistico-biologica, ignorando di fatto l'immensa area dei determinanti e dei processi sociali» (Seppilli 2014: 20). Il «terzo snodo» è un invito a relativizzare il punto di vista critico sulla biomedicina tenendo presente la condivisione dello stesso spazio storicoculturale che ha prodotto tanto il sapere scientifico biomedico quanto il sapere scientifico antropologico. 
8 L'interrelazione tra dimensione biologica - corpo, salute, malattia - e dimensione sociale - condizioni storico-culturali, politico-economiche, sanitarie - nella fisionomia specifica che assume in contesti situati, è una direttrice di analisi e riflessione che l'antropologia medica ha integrato nel suo paradigma interpretativo contemporaneo da diversi decenni. Fra gli innumerevoli contributi esistenti, a tale riguardo, se ne possono menzionare due ben rappresentativi, a puro titolo esemplificativo. Shahaduz Zaman (2005), medico e antropologo, in una ricerca etnografica in un reparto ortopedico di un ospedale del Bangladesh, ha rilevato che le condizioni di salute dei degenti erano esito diretto di specifiche caratteristiche sociopolitiche della più ampia società riguardo a gerarchie, disuguaglianze sociali, meccanismi della violenza, rapporti di forza e relazioni di genere. Per esempio, un quattordicenne aveva perso le gambe perché un altro giovane, in seguito a una discussione, lo aveva spinto giù da un treno; a un uomo erano state tagliate le mani da un parente per acquisire un appezzamento di terreno; gli esponenti di un gruppo politico avevano tagliato le membra ai militanti di un gruppo rivale per vendetta. Si tratta di episodi di violenza che rispecchiano una società in cui aggressioni, omicidi e fatti di sangue sono temi persistenti e realtà consuete della vita sociale ${ }^{6}$.

9 Margaret Lock, studiando il discorso biomedico sugli aspetti genetici della malattia di Alzheimer, ha portato l'attenzione sulla variabilità del rapporto tra una mutazione considerata fattore di rischio (ApoE $\varepsilon 4)^{7}$ e l'effettiva emersione della patologia in gruppi umani differenti. Presso i Pigmei e altre popolazioni con un'economia di sussistenza basata su caccia e raccolta, l'ApoE $\varepsilon 4$ sembra proteggere dalla malattia di Alzheimer. Presso alcuni gruppi della Nigeria, indipendentemente dalla presenza di questa mutazione, tale patologia ha livelli di incidenza minimi. Pare che in Nord America l'ApoE $\varepsilon 4$ favorisca l'insorgenza dell'Alzheimer fra gli afro-americani, ma in misura inferiore rispetto ai "bianchi". Di conseguenza, si deve supporre che in Africa intervengano elementi di riduzione del rischio, mentre in Nord America agiscono dei fattori che lo accrescono (Lock, Lloyd, Prest 2006; Lock et al. 2007; Lock 2013; Pasquarelli 2014). Quest'ultimo esempio mostra con chiarezza la complessità del rapporto causale tra salute, malattia e ambiente, rapporto che, in anni recenti, è stato riconosciuto anche nel campo dell'epigenetica: la comprensione del ruolo svolto dai geni nell'insorgenza di malattie deve essere situata entro la griglia delle interrelazioni costanti fra cofattori genetici, proteici e, appunto, ambientali.

Inoltre, tale attenzione verso le influenze dell'ambiente sociale ed ecologico che subiscono le cellule ha favorito il superamento delle visioni deterministiche di percorsi biologici causali lineari e unidirezionali: di fatto, l'impatto degli influssi esterni sulle cellule ricade sul funzionamento dei geni. Questo tipo di visione favorisce il dialogo tra una biomedicina critica e l'antropologia medica rispetto alla individuazione dei determinanti di salute, dei fattori di rischio, e della complessità delle relazioni causali che intercorrono fra di loro.

\section{Campi di causazione}

11 Nella riflessione su inquinamento industriale, ambiente e salute, tema egemone è rappresentato dai nessi causali tra emissioni contaminanti e la diffusione di patologie. $\grave{E}$ un rapporto "superficiale", non in quanto marginale, ma proprio in quanto evidente, in superficie, e compito dell'antropologia è restituire a questo legame la sua profondità 
svelandone l'irriducibilità a una deterministica linearità causa-effetto. Nei contesti industriali, le criticità sanitarie quali: diffusione di neoplasie, disfunzioni tiroidee, malformazioni neonatali, asma bronchiale, e tutte le altre condizioni di salute plausibilmente riconducibili alle emissioni inquinanti tendono a favorire la produzione e la diffusione di discorsi critici sull'eziologia stessa della malattia, i quali chiamano in causa le responsabilità delle compagnie industriali e delle istituzioni governative. Si innescano, in questo modo, frizioni (Tsing 2005) scientifiche, giuridiche, storiche e politiche che coinvolgono sistematicamente rappresentanti delle istituzioni pubbliche, magistrati, compagnie industriali, centri di ricerca, sindacati, movimenti per la giustizia ambientale e per la salute pubblica.

Come messo in evidenza già da Allan Young (1982) e più di recente, tra altri, da VinhKim Nguyen e Karine Peschard (2003), Paul Farmer (2003), Sharon Hamdy (2008) e Didier Fassin (2014), l'insorgenza di patologie deve essere indagata lungo la linea di un'eziologia socio-bio-politica capace di cogliere e analizzare le confluenze tra patogenesi, fattori storico-culturali, socioeconomici, politici e il corpo.

Questo quadro teorico richiama il carattere fisico-politico del binomio salute/malattia, la cui problematizzazione apre spazi di dialogo transdisciplinare con le posizioni più avanzate di un'epidemiologia critica che ha abbandonato con decisione gli essenzialismi deterministico-statistici in favore di modelli stocastici in cui alle cause unidirezionali si sostituisce, come sostenuto da Paolo Vineis (1990: 24), «una pluralità di reti di causazione». Inoltre, la ricerca etnografica, con la sua tensione verso la dimensione concreta ed esperienziale dei fenomeni, permette di cartografare (Deleuze 2007) le politeticità causali e di esplorarne le reti di causazione all'interno dei rapporti di forza che le determinano, le veicolano e le manipolano. Lo studio dei processi di fabbricazione e negoziazione dei nessi tra inquinamento industriale e patologie nella città di Brindisi, la quale è dominata da forti tensioni tra compagnie industriali ed energetiche, istituzioni pubbliche e movimenti per la giustizia ambientale, ha dimostrato che la molteplicità delle relazioni che di volta in volta determinano definizione e interpretazione del rapporto tra presenza industriale e patologie si situa all'interno di "campi di causazione" (Ravenda 2018: 34-42). Si tratta di spazi relazionali in cui i nessi causali sono oggetto di dinamiche conflittuali che producono un costante turbamento generativo e uno scambio sistematico tra i corpi "esposti" alle emissioni inquinanti, con le loro capacità trasformative di agency e di advocacy, e i dispositivi giuridici, storici, biomedici, politici strutturanti e oggettivanti che presiedono alla produzione delle classificazioni.

Da questa prospettiva teorico-metodologica esplorare i nessi tra inquinamento e malattia significa attuare una continua riconfigurazione della causalità che tenga conto della ramificazione temporale delle sue variabili co-agenti poiché, piuttosto, il "problema diventa chiedersi che cosa stia a monte: che cosa ha innescato il meccanismo patogenetico, che cosa ha portato l'esposizione dell'individuo all'agente patogeno, che cosa ha consentito a quest'ultimo di dare avvio al processo patologico» (Vineis 1990: 51). Per penetrare questa complessità occorre investigare l'insieme dei fattori concomitanti storico-politici, delle condizioni e delle disuguaglianze sociali e socioeconomiche che delimitano, circoscrivono e attraversano i campi di causazione. In altri termini, significa prendere in considerazione le scelte politiche di sviluppo dei territori e di sviluppo industriale, le normative vigenti di controllo delle soglie di emissione e l'eventuale mancato rispetto delle stesse, i conflitti di interesse degli 
istituti e degli enti di ricerca, le contese giuridiche sul diritto alla salute e sulle responsabilità delle compagnie industriali, le contraddizioni nei programmi di promozione della sostenibilità energetica (Franquesa 2018), e così via.

Un altro aspetto della questione è rappresentato dalle ricadute delle attività degli impianti inquinanti sul territorio, sulla sua economia e sulle politiche culturali di promozione turistica; non solo nei termini ovvi di segni dell'impatto industriale su paesaggi, ambienti naturali, prodotti agro-alimentari locali, a detrimento, insomma, del marketing territoriale. Infatti, come mostrato da Tamara Steger (2009) nel suo studio sui movimenti per la giustizia ambientale nella Lituania post-sovietica, se quegli stessi segni, da un lato, sono prove tangibili del degrado ambientale e dell'inquinamento indotti dalle industrie, dall'altro possono diventare, attraverso la loro esibizione, dei vettori per la creazione di un senso di appartenenza e di un sentimento di località radicato nella costruzione di un passato pre-industriale "idilliaco" con il rischio di una deriva identitaria e nostalgica. Dopotutto, come messo in evidenza da Rob Nixon (2011) nel suo importante studio sulle mutazioni ambientali dovute all'inquinamento prodotto dalle attività umane, il tempo si costituisce come una componente determinante per la comprensione del livello di danno fisico che una sostanza tossica può provocare. Lo studioso sintetizza questi processi con l'espressione slow violence: «una violenza che si verifica gradualmente e invisibilmente, una violenza dalla distruttività ritardata dispersa nel tempo e nello spazio, una violenza logorante che in genere non è vista affatto come violenza» (Nixon 2011: 2).

Violenza, tempo, politeticità causale sono elementi costitutivi e di manipolazione di "campi di causazione" sovrapposti che sembrano primariamente costruiti intorno al tema dell'inquinamento e della malattia e i quali, invece, nei diversi posizionamenti delle forze istituzionali e degli attori sociali, si rivelano variamente declinati rispetto alle retoriche del passato preindustriale, a un presente caratterizzato da problemi ambientali, economici, sanitari e a un futuro "diverso", sostenibile, da progettare e fondare sulla valorizzazione dei patrimoni culturali e naturali locali.

\section{L'incorporazione delle eziologie politiche}

Le prospettive di studio e analisi dell'antropologia su inquinamento e salute, nonostante l'eterogeneità degli approcci e dei contesti di ricerca, convergono sul riconoscimento della relazione che sempre intercorre tra corpo e Stato e ne hanno evidenziato le molteplici forme di espressione e manifestazione. Gli aspetti politici dell'incorporazione (Csordas 1990) implicano la considerazione simultanea dei processi di salute/malattia e dei più ampi processi sociopolitici dotati del potere di inscriversi sui corpi, nonché la considerazione «dell'intimità dei poteri statali su forme diverse di esperienza corporea, come la nazionalità, la cittadinanza, la scienza, la violenza, la malattia» (Pizza, Johannessen 2009: 20). All'interno dei campi di causazione, queste relazioni scaturiscono dalla specificità dei rapporti tra corpi e istituzioni, e la loro articolazione oltrepassa il momento patogenetico per sfociare in vere e proprie "eziologie politiche" ${ }^{8}$ che evidenziano il modo in cui i livelli di corruzione, di conflitto di interesse e di diseguaglianza socioeconomica sono collegati con i peggiori indici di salute in un'intera società (Nguyen, Peschard 2003). Ciò risulta patente quando l'insorgenza di una malattia è in strettissima relazione con la presenza, per esempio, di istituzioni corrotte cui imputare la responsabilità diretta per l'inquinamento di acqua $\mathrm{e}$ 
di aria, per la cattiva gestione dei rifiuti tossici, per i livelli incontrollati di emissioni industriali e la commercializzazione di cibo non sicuro o contaminato. La malattia, cioè, è qui il risultato dell'inscrizione nei corpi di forze sociali e politiche storicamente determinate, dell'incorporazione delle disuguaglianze sociopolitiche e delle disparità tanto nell'accesso alle risorse per la salute quanto nell'esposizione ai fattori di rischio.

18 I saggi contenuti in questo volume rappresentano ciascuno a suo modo linee di attraversamento dei campi di causazione affrontando, da diverse prospettive interpretative e in riferimento a contesti plurali, il rapporto tra ambiente e tutela della salute laddove la presenza di fattori di rischio ambientale/industriale attivano risposte, talora confliggenti, da parte di attori sociali portatori di interessi diversificati. Alcuni dei temi che caratterizzano trasversalmente i contributi sono fra i pilastri teoretici del dibattito su inquinamento e salute/malattia, quali le dinamiche sociopolitiche che si dispiegano sui territori e le comunità locali interessate, la loro incorporazione, anche nella forma di differenze nell'esposizione alle fonti di inquinamento; la costruzione della memoria - che è anche, sempre, una memoria corporea - e di un sapere che oscillano tra adesione e contestazione di quei saperi capaci di disciplinare memoria, conoscenza ed esperienza vissuta: quello biomedico, quello politico, quello trasmesso dalle industrie; e, ancora, le contraddizioni che sferzano i campi di causazione, con un valido esempio in quelle sollevate da progetti di riconversione green.

Ad aprire il volume, il contributo di Enzo V. Alliegro riflette sul complesso sistema di produzione e diffusione dei dati scientifici e di monitoraggio delle emissioni inquinanti in contesti a forte presenza industriale. La ricerca si concentra sul caso paradigmatico della città di Taranto dove il più grande stabilimento siderurgico a ciclo continuo d'Europa, attivo già dai primi anni Sessanta del secolo scorso, con un impatto ambientale che risulta ai massimi livelli di rischio sanitario, rappresenta un contesto privilegiato per osservare le dinamiche conflittuali del rapporto tra inquinamento industriale e diffusione di patologie. L'approccio di studio di carattere storicoantropologico consente di cogliere la dimensione mutevole della percezione della crisi ambientale quale costrutto socioculturale, agente e agito, che risente dello sviluppo dei sistemi normativo-giuridici, scientifico-tecnologici e degli assetti culturali, sociali e politico-economici.

20 La presenza industriale può trasformare la percezione della popolazione locale del proprio rapporto con il territorio come dimostra il caso di Viggiano (Basilicata), interessato dall'attività di estrazione di idrocarburi, esaminato da Maria Luisa Matera. L'analisi etnografica si è soffermata sulla criticità di un legame in crisi, nella sua riarticolazione nei contenuti e nei significati dei concetti di salute/malattia e "normalità" a livello individuale e socioculturale. Le posizioni e le opinioni di diversi attori sociali - gli esperti, i militanti, gli opinion leaders che comunicano con la stampa, la stessa popolazione - agiscono su tali definizioni esponendo le categorie di rischio, salute, malattia a molteplici declinazioni. Così, per esempio, la "malattia" (cancro) diventa, percettivamente, la presentificazione di una catastrofe che la comunità non accetta, e la malattia si fa, addirittura, preferibile alla mancanza di lavoro. La salute, invece, diventa un dominio esclusivamente individuale, sottratto a ogni gestione collettiva, nonostante la presenza attiva di persone disposte ad attuare delle possibili alternative per stimolare la comunità.

21 Francesco Danesi ha offerto una declinazione ulteriore delle dinamiche del rapporto tra rischio ambientale, territorio, salute, focalizzandosi sulle conseguenze politico- 
economiche, coi loro risvolti sociali e individuali, nelle aree terremotate del Centro Italia. La presenza-assenza dello Stato nella fase emergenziale, con le sue retoriche e narrative, si esplicita in spazi in cui i programmi istituzionali di assistenza e intervento non sempre rispondono alle esigenze reali della popolazione, che piuttosto subisce e denuncia una "strategia dell'abbandono". Qui si apre una zona grigia in cui il rapporto tra le persone e l'ambiente assume connotati tecnocentrici e l'esercizio del potere grava soprattutto sulle frange più vulnerabili delle popolazioni locali con dei processi di plasmazione e riformulazione della memoria locale che occultano gli interessi politicoeconomici delle strategie della ricostruzione.

Agata Mazzeo, a fronte di un'etnografia di lungo periodo svolta in Italia e in Brasile, affronta il tema dell'attivismo e dei disastri connessi al mercato globale dell'amianto a partire dal ruolo che in queste dinamiche ha il corpo delle persone: un corpo che non è soltanto il luogo in cui si mostra l'impatto del disastro amianto-correlato, ma un corpo capace di esprimere la propria agentività in un uso politico strategico da parte degli attivisti. Questi ultimi, infatti, usano il corpo quale sede di sapere e di memoria per negoziare la tutela della salute pubblica e contrastare le dinamiche globali e i processi socioculturali e politico-economici che percepiscono e interpretano come forme di ingiustizia sociale.

Conclude il dossier Andrea F. Ravenda che si concentra sull'analisi dei conflitti locali connessi alla presenza di un impianto di raffinazione di petrolio a Milazzo, nella Sicilia nord-occidentale. A partire da un'esperienza etnografica condotta a stretto contatto con alcuni lavoratori della raffineria e con alcuni militanti dei movimenti per la giustizia ambientale, il contributo evidenzia come le valutazioni pubbliche sull'intreccio tra presenza industriale, ambiente e salute che si definiscono all'interno di conflittuali "campi di causazione" producano vere e proprie frizioni nei nuclei famigliari dei lavoratori. Da questa prospettiva le famiglie e le stesse biografie dei lavoratori rappresentano spazi di attrito utili per comprendere il più complesso sistema che connette le istanze globali del rischio ambientale con le specificità locali e con le esperienze delle persone che vivono e lavorano in aree industriali.

\section{BIBLIOGRAFIA}

Ackerknecht E. H., 1971 Medicine and Ethnology: Selected Essays, Johns Hopkins University Press, Baltimore.

Alliegro E. V., 2012 Il totem nero. Petrolio, sviluppo e conflitti in Basilicata, CISU, Roma.

Atkinson P., 1987 «The Effectiveness of Shamans in an Indonesian Ritual», in American Anthropologist, 89: 342-355.

Baer H., Singer M., Johnsen J., 1986 «Toward a critical medical anthropology», in Social Science and Medicine, 23: 95-98.

Balshem M., 1993 Cancer in the Community: Class and Medical Authority, Smithsonian Institution Press, Washington. 
Benadusi M., Revet S., 2016 «Disaster trials: a step forward», in M. Benadusi, Revet S. (a cura di), On the Witness Stand: Environment Crises, Disasters and Social Justice, in Archivio Antropologico Mediterraneo, 18: 7-15.

Beck U., 1987 «The Anthropological Shock: Chernobyl And The Contours Of The Risk Society», in Berkeley Journal of Sociology, 32: 153-165.

Biehl J., 2013 «The judicialization of biopolitics: Claiming the right to pharmaceuticals in Brazilian courts», in American Ethnologist, 40: 419-436.

Biehl J., Petryna A. (eds), 2013 People come First. Critical Studies in Global Health, Princeton University Press, Princeton and Oxford.

Boudia S., Jas N. (eds), 2014 Powerless Science? Science and Politics in a Toxic World, Berghahn Books, New York-Oxford.

Conti L., 1977 Visto da Seveso. L'evento straordinario e l'ordinaria amministrazione, Feltrinelli, Milano.

Csordas T., 1990 «Embodiment as a Paradigm for Anthropology», in Ethos. Journal of the Society for Psychological Anthropology, 18: 5-47.

Deleuze G., 2007 Che cos'è un dispositivo?, Cronocopio, Napoli.

Del Vecchio-Good M. J., 1995 «Cultural studies of biomedicine: an agenda for research», in Social Science and Medicine, 41: 461-473.

de Martino E., 1958 Morte e pianto rituale nel mondo antico. Dal lamento pagano al pianto di Maria, Einaudi, Torino.

de Martino E., 1961 La terra del rimorso. Contributo a una storia religiosa del Sud, Il Saggiatore, Milano.

Ember C. R., Ember M., 2004 Encyclopedia of Medical Anthropology. Health and Illness in the world's cultures, Kluwer Academic-Plenum Publishers, New York.

Fassin D., 2014 «Cinque tesi per un'antropologia medica critica», in AM. Rivista della Società italiana di antropologia medica, 37: 33-50.

Foster G. M., Anderson B. G., 1978 Medical anthropology, Wiley, New York.

Franquesa J., 2018 Power Struggles. Dignity, Value, and the renewable energy frontier in Spain, Indiana University Press, Bloomington.

Gordon D., 1988 «Tenacious assumptions in Western Medicine», in M. Lock, Gordon D. (eds), Biomedicine examined, Kluwer Academic Publishers, Dordrecht-Boston-London: 19-56.

Hanh R. A., Gaines A. D. (eds), 1985 Physician of Western Medicine. Anthropological Approaches to Theory and Practice, D. Reidel Publishing Company, Dordrecht-Boston-Lancaster.

Hahn R. A., Inhorn M. C. (eds), 2009 Anthropology and Public Health: Bridging Differences in Culture and Society, Oxford University Press, New York.

Hamdy S. F., 2008 «When the state and sour kidneys fail: Political etiologies in an Egyptian dialysis ward», in American Ethnologist, 35: 553-569.

Johnson T. M., Sargent C. F. (eds), 1990 Medical Anthropology. Contemporary theory and method, Praeger, Westport.

Khare R. S., 1987 «The Bhopal Industrial Accident: Anthropological and Civic Issues», in Anthropology Today, 3: 4-6. 
Lindenbaum S., Lock M. (eds.), 1993 Knowledge, power, and practice. The anthropology of medicine and everyday life, University of California Press, Berkley-Los Angeles-London.

Lock M., 2013 The Alzheimer Conundrum. Entanglements of Dementia and Aging, Princeton University Press, Princeton.

Lock M., Gordon D. (eds), 1988 Biomedicine Examined, Kluwer Academic Publishers, DordrechtBoston-London.

Lock M., Lloyd S., Prest J., 2006 «Genetic Susceptibility and Alzheimer's Disease. The Penetrance and Uptake of Genetic Knowledge», in A. Leibing, Cohen L. (eds), Thinking about dementia. Culture, loss, and the anthropology of senility, Rutgers University Press, New Brunswich: 123-154.

Lock M., Freeman J., Chilibeck G., Beveridge B., Padolsky M., 2007 «Susceptibility Genes and the Question of Embodied Identity», in Medical Anthropology Quarterly, 21: 256-276.

Lock M., Nguyen V. K., 2010 An anthropology of Biomedicine, Wiley-Blackwell, Oxford.

Nguyen V.-K., Peschard K., 2003 «Anthropology, Inequality, and Disease: A Review» in Annual Review of Anthropology, 32: 447-474.

Nixon R., 2011 Slow violence and the environmentalism of the poor, Harvard University Press, Cambridge, Massachusetts-London.

Pasquarelli E., 2008 «L'etnografia dell'ospedale. Alcune prospettive di studio nel dibattito antropologico contemporaneo», in AM. Rivista della Società Italiana di Antropologia Medica, 21-26: 475-496.

Pasquarelli E., 2014 «Esplorare un enigma. La malattia di Alzheimer tra dilemmi diagnostici, progetti di prevenzione e linee di ricerca nel campo della genetica», in AM. Rivista della Società Italiana di Antropologia Medica, 37: 663-667.

Pasquarelli E., 2018 Antropologia dell'Alzheimer. Neurologia e politiche della normalità, Alpes Italia, Roma.

Petryna A., 1998 «A Technical Error: measures of Life after Chernobyl», in Social Identities, 4: 73-92.

Petryna A., 2002 Life Exposed: Biological Citizens after Chernobyl, Princeton University Press, New York-Princeton.

Pfeiderer B., Bibeau G. (eds), 1991 Anthropologies of Medicine. A colloquium on West European and North American Perspectives, Vieweg, Heidelberg.

Pizza G., Johannessen H., 2009 «Editorial. Two or three things about Embodiment and the State», in G. Pizza, Johannessen H. (eds), Embodiment and the State. Health, biopolitics and the intimate life of State powers, in AM. Rivista della Società italiana di antropologia medica, 27-28: 13-20.

Pizza G., Ravenda A. F. (a cura di), 2012 Presenze internazionali. Prospettive etnografiche sulla dimensione fisico-politica delle migrazioni in Italia, in AM. Rivista della Società italiana di antropologia medica, 33-34, numero monografico.

Prato G., 1993 «Political decision-making: environmentalism, ethics and popular participation in Italy», in K. Milton, Environmentalism. The view from Anthropology, Routledge, London-New York: 173-186.

Ravenda A. F., 2014 «"Ammalarsi di carbone”. Note etnografiche su salute e inquinamento industriale a Brindisi», in AM. Rivista della Società italiana di antropologia medica, 38: 615-633. Ravenda A. F., 2018 Carbone. Inquinamento industriale, salute e politica a Brindisi, Meltemi, Milano. 
Rhodes L. A., 1990 «Studying Biomedicine as a Cultural System» in T. M. Johnson, Sargent C. F. (eds), Medical Anthropology. Contemporary theory and Method, Praeger, Westport: 159-173.

Seppilli T., 1968 «Intervento» in La medicina e la società contemporanea, Editori Riuniti, Roma: 240-243.

Seppilli T., 1996 «Antropologia Medica: fondamenti per una strategia», in AM. Rivista della Società italiana di antropologia medica, 1-2: 7-22.

Seppilli T., 2008a Scritti di Antropologia Culturale I. I problemi teorici, gli incontri di culture, il mondo contadino, a cura di M. Minelli, Papa C., Leo S. Olschki, Firenze.

Seppilli T., 2008b Scritti di Antropologia Culturale II. La festa, la protezione magica, il potere, a cura di M. Minelli, Papa C., Leo S. Olschki, Firenze.

Seppilli T., 2014 «Antropologia medica e strategie per la salute», in AM Rivista della Società italiana di antropologia medica, 37: 17-32.

Steger T., 2009 «The Role of Culture and Nationalism in Latvian Environmentalism and the Implications for Environmental Justice», in J. Agyeman, Ogneva-Himmelberger Y. (eds), Environmental Justice and Sustainability in the Former Soviet Union, The MIT Press, Cambridge: 141-152.

Tsing A., 2005 Frictions. An Anthropology of Global connection, Princeton University Press, Princeton. Vineis P., 1990 Modelli di rischio. Epidemiologia e causalità, Einaudi, Torino.

Waldman L., 2011 The Politics of Asbestos: Understandings of Risk, Disease and Protest, Routledge, London.

Young A., 1982 «The Anthropologies of Illness and Sickness», in Annual Review of Anthropology, 11: 257-285.

Zaman S., 2005 Broken limbs, broken lives. Ethnography of a hospital ward in Bangladesh, Het Spinhuis, Amsterdam.

\section{NOTE}

1. Concepito in comune dai due Autori, questo testo è stato scritto E. Pasquarelli per i paragrafi 2 e 4 e da A. F. Ravenda per i paragrafi 1 e 3.

2. Per una panoramica dell'approccio dell'antropologia medica a tali tematiche, cfr. Ember, Ember 2004: 26, 271, 973.

3. Per un dettagliato resoconto dell'evento di Seveso cfr. Conti 1977; sul disastro di Chernobyl, cfr. Beck 1987 e Petryna 1998; su quello di Bhopal, cfr. Khare 1987, per l'esplosione di Brindisi cfr. Prato 1993, Ravenda 2018.

4. I saggi che compongono questa sezione monografica sono l'esito di una selezione dei principali interventi del convegno di Perugia distribuiti tra le principali riviste italiane di Antropologia; in questo caso, si tratta di quelli provenienti dalla sessione "Salute, Ambiente, Rischio". Desideriamo ringraziare il Presidente della SIAM, Alessandro Lupo, e il Direttore della rivista AM, Giovanni Pizza, per averci dato la possibilità di selezionare i contributi dall'ampia disponibilità del convegno. Allo stesso tempo desideriamo ringraziare la Direttrice di Archivio Antropologico Mediterraneo, Gabriella D'Agostino, e la redazione della rivista per aver accettato la proposta editoriale.

5. Per quel che concerne la storia degli studi antropologici sulla biomedicina si propone una rassegna cui fare generale riferimento: Hahn, Gaines 1985; Gordon 1988; Lock, Gordon 1988; 
Johnson, Sargent 1990; Rhodes 1990; Pfeiderer, Bibeau 1991: 121-181; Lindembaum, Lock 1993; Del Vecchio-Good 1995; Seppilli 1996.

6. Cfr. Pasquarelli 2008: 480-481 e relativa bibliografia.

7. Nel 1996 è stato scoperto che una modificazione del quarto allele del gene polimorfico apoliproteina E (ApoE), del cromosoma 19, poteva essere in relazione all'insorgenza dell'Alzheimer tardivo. Fra le varianti genetiche dell'apoliproteina $\mathrm{E}$, cioè $\varepsilon 2$, ع3, ع4, solo quest'ultima è considerata un fattore di rischio, mentre la modifica del secondo allele sembra in rapporto a una sua diminuzione (Pasquarelli 2018: 62).

8. Per un'analisi del concetto di "eziologia politica" cfr. lo studio di Sharon Hamdy (2008) sulla diffusione delle patologie renali tra le classi meno abbienti in Egitto.

\section{RIASSUNTI}

I saggi contenuti in questo dossier sono l'esito di un confronto pubblico sull'intreccio tra salute, ambiente e rischio emerso da una sessione tematica focalizzata su questo tema del $2^{\circ}$ convegno nazionale della Società Italiana di Antropologia Medica (Perugia 14-16 giugno 2018) dedicato alla memoria del fondatore Tullio Seppilli. Gli autori affrontano da diverse prospettive interpretative e in riferimento a contesti plurali, il rapporto tra ambiente e tutela della salute e i modi in cui la presenza di fattori di rischio ambientale/industriale attiva risposte, talora confliggenti, da parte di attori sociali portatori di interessi diversificati. In un tale quadro di complessità, il dossier ospita contributi teorici e ricerche etnografiche che esplorano, nella loro molteplicità, le articolazioni globali della crisi ambientale con le specificità locali incarnate nelle esperienze delle persone che vivono, lavorano, si ammalano in territori danneggiati o contaminati.

The papers result from a public debate on the intertwining of health, environment and risk as emerged from a panel focused on these topics of the 2nd national conference of the Italian Society of Medical Anthropology (Perugia June 14-16, 2018) dedicated to the memory of its founder Tullio Seppilli. The authors discuss - within different interpretative perspectives and in reference to plural contexts - the relationship between environment and health protection and the ways in which the presence of environmental and/or industrial risk factors stimulate sometimes conflicting responses in social actors with diversified interests. In such a complex framework, this special issue hosts theoretical contributions and ethnographic researches that, in their multiplicity, explore the global articulations of the environmental crisis with its local peculiarities embodied in the experiences of the people who live, work and fall ill in territories damaged or contaminated.

\section{INDICE}

Parole chiave : antropologia medica, ambiente, rischio, causalità, salute

Keywords : medical anthropology, environment, risk, causality, health 


\section{AUTORI}

\section{ELISA PASQUARELLI}

Dipartimento di Filosofia, Scienze Sociali, Umane e della Formazione, Università degli Studi di Perugia elisa.pasquarelli@gmail.com

\section{ANDREA FILIPPO RAVENDA}

Dipartimento Storia Culture Civiltà, Università di Bologna andrea.ravenda@unibo.it 